\title{
AVALIAÇÃO DA PARASITEMIA EM MULHERES PORTADORAS DE INFECÇÃO PELO TRYPANOSOMA CRUZI DURANTE E APÓS A GESTAÇÃO
}

\author{
Carlos A.S. Menezes, Achiléa L. Bitterncourt, Eduardo Mota, \\ Italo Sherlock e Janete Ferreira
}

\begin{abstract}
Avaliou-se o perfil parasitêmico de 119 chagásicas crônicas, através de 828 xenodiagnósticos, realizados durante (465 xenos) $e$ após a gravidez (363 xenos) visando rastrear uma possivel variaçâo da parasitemia nesses periodos. A freqüência dexenos positivos foi maior durante a gestação. Por outro ládo, a frequiencia de triatomíneos infectados foi, também, maior durante a gravidez, indicando niveis parasitêmicos mais elevados neste periodo. Apenas $17 \%$ das mães estudadas tiveram dois ou mais xenos positivos durante a gravidez. Nestas mulheres, a diferença entre a freqüencia de xenos positivos durante e após a gravidez foi elevada, sugerindo ter havido exacerbação da infecção chagásica, pelo menos em algumas delas.
\end{abstract}

Palavras-chaves: Xenodiagnóstico. Parasitemia na gestação. Doença de Chagas na gravidez. Doenças infecciosas e gravidez.

$\mathrm{Na}$ fase aguda da doença de Chagas, a parasitemia é elevada e persistente, diminuindo à medida que o paciente evolui para a fase crônica, quando é intermitente e permanece em níveis muito baixos, somente podendo ser detectada pelo xenodiagnóstico (xeno) e/ou cultura. Reativação da infecção aguda tem sido observada em pacientes imunodeprimidos pelo uso de drogas ou na sindrome da imunodeficiência adquirida (AIDS) ${ }^{712}$.

$\mathrm{Na}$ gravidez ocorrem modificações da imunidade mediada por células, o que torna a gestante mais susceptível às infecções ${ }^{3}$. Na hanseníase, por exemplo, há, com freqüência, agravamento da doença durante a gravidez, com acentuada melhora após o parto 5 . Com referência à doença de Chagas, raros são os estudos acerca de sua evolução na gestação.

Storni \& Bolsi ${ }^{10}$, avaliando um grupo de 50 gestantes chagásicas, através de testes sorológicos e de xenos, ecomparando-o com um grupo controle

\footnotetext{
Trabalho da Maternidade Climério de Oliveira, Departamentos de Patologia e de Medicina Preventiva da Faculdade de Medicina, Universidade Federal da Bahia, do Serviço de Entomologia do Centro de Pesquisas Gonçalo Moniz/ FIOCRUZ e do Setor de Imunologia do Laboratório Central do Estado da Bahia, Salvador, BA.

Endereço para correspondencia: Dr. Carlos A.S. Menezes R. Pacífico Pereira 406/1003, 40100-170 Salvador, BA Recebido para publicação em $10 / 12 / 91$.
}

de 40 chagásicas não grávidas, de mesma faixa etária, observaram que em algumas grávidas havia maior freqüência de xenos positivos e quadro sorológico sugestivo de reativação da infecção. Por outro lado, Szarfman e cols ${ }^{11}$ pesquisando o IgM sérico específico em 28 gestantes chagásicas, encotraram positividade em apenas seis. Recentemente, Reyes e cols ${ }^{9}$ estudando 20 gestantes chagásicas detectaram IgM específico em $50 \%$ delas e apenas estas transmitiram sua infecção ao concepto. No entanto, Biocca \& Sequeira ${ }^{2}$, aplicando xenos seriados em chagásicas grávidas e não grávidas, constataram maior frequiência de positividade nas últimas. Experimentalmente, já se observou um discreto aumento na intensidade da parasitemia em fêmeas grávidas ${ }^{8}$. No entanto, Carlier e cols ${ }^{4}$ concluíram que a gravidez não influencia na infecção murina pelo Trypanosoma cruzi.

O objetivo deste trabalho é avaliar o comportamento da parasitemia durante a gestação na doença de Chagas, através da realização de xenos, utilizando como controle o mesmo grupo de mulheres, após o parto.

\section{MATERIAL E MÉTODOS}

No serviço de atenção pré-natal da Maternidade Climério de Oliveira da Universidade Federal da 
Menezes CAS, Bittencourt AL, Mota E, Sherlock I, Ferreira J. Avaliação da parasitemia em mulheres portadoras de infeç̧ão pelo Trypanosoma cruzi durante e após a gestação. Revista da Sociedade Brasileira de Medicina Tropical 25:109-113, abr-jun, 1992.

Bahia, foram selecionadas 160 mulheres oriundas do interior do estado, todas na fase crônica da doença de Chagas, com positividade concomitante para as reações de imunofluorescência indireta antiT. cruzi e de fixação de complemento (GuerreiroMachado). Excluíram-se as pacientes que tinham visitado zona endêmica ou que tinham recebido hemotransfusão recente, para evitar possíveis interferências na parasitemia.

As gestantes foram submetidas a xenos, a partir da $12^{\text {a }}$ semana, com intervalos de duas a quatro semanas, até ao termo. Igual procedimento foi reiniciado a partir da segunda semana de puerpério até, no máximo, à $42^{a}$ semana. Os exames foram feitos sempre no horário entre 14 e 16 horas. Para cada caixa de xeno, foram utilizadas 10 ninfas do $5^{\circ}$ estágio de Triatoma infestans, criadas em laboratório. Trinta dias após a aplicação do xeno, foi feita a pesquisa de $T$. cruzi, individualmente, mediante dissecção do inseto. $O$ conteúdo intestinal foi misturado com salina examinado ao microscópio com aumento de $400 \mathrm{X}$. A intenção era a de realizar igual número de xenos por paciente, antes e depois da gestação, num total de seis a oito exames.

Para avaliar o comportamento da parasitemia durante e após a gravidez, foram consideradas a freqüência de xenos positivos em cada fase e a freqüência de triatomíneos positivos nos xenos realizados antes e depois da gestação. A freqüência da parasitemia foi avaliada pelo percentual de xenos positivos. Por outro lado, os níveis parasitêmicos foram aferidos de acordo com a freqüência de triatomíneos positivos no total dos exames feitos em cada fase. Nas pacientes que fizeram, pelo menos, um xeno no segundo e terceiro trimestres, realizou-se também o estudo comparativo dos resultados obtidos. Para testar diferenças entre as proporções, aplicou-se o teste do Qui-quadrado para amostras independentes.

\section{RESULTADOS}

Foram triadas 160 mulheres na fase crônica da doença de Chagas, admitidas a partir da $12^{\mathrm{a}}$ semana de gestação, no período de maio de 1984 a outubro de 1987. Deste grupo, excluíram-se 41 pacientes que abandonaram o protocolo, tendo sido avaliado um total de 119 gestantes. A média das idades foi de 30,9 anos. Por motivos diversos, não foi possível obter sempre um número igual de xenos antes e após a gestação e, por vezes, as pacientes fizeram menos de três exames em cada período. Por isso, para a análise comparativa dos resultados, as 119 pacientes foram agrupadas de três modos diferentes, na dependência do número de xenos feitos durante $\mathrm{e}$ após gestação:

Grupo I - Constituido de 119 mulheres com pelo menos um exame realizado durante e após a gestação. Estas pacientes fizeram, em média, quatro xenos durante a gestação, variando de um a sete e, em média, três xenos após, variando de um a seis. Pode-se observar que hove aumento estatisticamente siginificante na freqüência de xenos e de triatomíneos

\section{Tabela 1 - Resultados do grupo de 119 mulheres com doença de Chagas crônica, com ao menos um exame realizado durante e após a gravidez (grupo I).}

\begin{tabular}{|c|c|c|c|}
\hline \multicolumn{2}{|c|}{ Durante a gestação } & \multicolumn{2}{|c|}{ Após a gestação } \\
\hline Total & Positivos & Total & Positivos \\
\hline & $n^{\circ}$ & & $n^{\circ}$ \\
\hline
\end{tabular}

Significância estatística

Xenos

realizados

465

$91 \quad 19,6$

363

48

13,2

$\mathrm{x}_{1}^{2}=5,87 ; \mathrm{p}<0,05^{*}$

Triatomíneos

examinados

4646

224

4,8

3377

117

3,5

$\mathrm{x}_{1}^{2}=7,89 ; \mathrm{p}<0,05^{*}$

* Teste do Qui-quadrado para amostras independentes. 
Menezes CAS, Bittencourt AL, Mota E, Sherlock I, Ferreira J. Avaliação da parasitemia em mulheres portadoras de infecção pelo Trypanosoma cruzi durante e após a gestação. Revista da Sociedade Brasileira de Medicina Tropical 25:109-113, abr-jun, 1992.

Tabela 2 - Resultados obtidos em 96 mulheres com doença de Chagas crônica e que fizeram dois ou mais xenos durante e após a gestação (grupo II).

\begin{tabular}{|c|c|c|c|c|c|c|c|}
\hline & \multicolumn{3}{|c|}{ Durante a gestação } & \multicolumn{3}{|c|}{ Após a gestação } & \multirow{3}{*}{ Significância estatística } \\
\hline & \multirow[t]{2}{*}{ Total } & \multicolumn{2}{|c|}{ Positivos } & \multirow[t]{2}{*}{ Total } & \multicolumn{2}{|c|}{ Positivos } & \\
\hline & & $n^{o}$ & $\%$ & & $n^{\circ}$ & $\%$ & \\
\hline $\begin{array}{l}\text { Xenos } \\
\text { realizados }\end{array}$ & 400 & 75 & 18,8 & 329 & 40 & 12,2 & $\mathrm{x}_{1}^{2}=5,49 ; \mathrm{p}<0,05^{*}$ \\
\hline $\begin{array}{l}\text { Triatomíneos } \\
\text { examinados }\end{array}$ & 3946 & 175 & 4,4 & 3203 & 106 & 3,3 & $x_{1}^{2}=5,93 ; p<0,05^{*}$ \\
\hline
\end{tabular}

* Teste do Qui-quadrado para amostras independentes.

positivos durante a gravidez (Tabela 1). Neste grupo, apenas 20 mulheres $(17 \%)$ apresentaram dois ou mais xenos positivos durante a gravidez. Dos 90 xenos a que se submeteram durante a gestação, $70 \%$ foram positivos, enquanto que dos 64 exames realizados após a gestação, apenas $41 \%$ deram resultados positivos. A freqüência de triatomíneos infectados nestas 20 mães variou de $18 \%$ (antes) para $12 \%$ (depois).

Grupo II - Constituído de 94 mulheres selecionadas dentre as 119 do grupo I, porque fizeram dois ou mais exames em cada período. Foram aplicados, em média, quatro xenos durante a gestação, variando de dois a sete e, em média, três xenos após a gestação, variando de dois a seis. Neste grupo, a freqüência de positividade dos xenos edos triatomíneos foi também maior durante a gravidez com diferenças estatisticamente significantes (Tabela 2).

Grupo III - Constituído de 72 mulheres selecionadas dentre as 94 do grupo II, porque fizeram três ou mais xenos durante e após a gestação, tendo sido realizados, em média, quatro exames durante a gestação, variando de três a sete e quatro depois da gestação, variando de três a seis. Verificou-se, também, elevação da freqüência de positividade dos xenos e dos triatomíneos durante a gravidez, mas não houve diferenças estatisticamente significantes em relação ao período pós-parto (Tabela 3).

Tabela 3 - Resultados do grupo de 72 mulheres com doença de Chagas crônica, com três ou mais exames realizados durante e após a gestaçâo (grupo III).

\begin{tabular}{|c|c|c|c|c|c|c|c|}
\hline \multirow{3}{*}{$\begin{array}{r} \\
\\
\end{array}$} & \multicolumn{3}{|c|}{ Durante a gestação } & \multicolumn{3}{|c|}{ Após a gestação } & \multirow{3}{*}{ Significância estatística } \\
\hline & \multirow{2}{*}{ Total } & \multicolumn{2}{|c|}{ Positivos } & \multirow[t]{2}{*}{ Total } & \multicolumn{2}{|c|}{ Positivos } & \\
\hline & & $n^{\circ}$ & $\%$ & & $\mathrm{n}^{\circ}$ & $\%$ & \\
\hline $\begin{array}{l}\text { Xenos } \\
\text { realizados }\end{array}$ & 318 & 55 & 17,3 & 280 & 36 & 12,8 & $x_{1}^{2}=2,27 ; p>0,05^{*}$ \\
\hline $\begin{array}{l}\text { Triatomíneos } \\
\text { examinados }\end{array}$ & 3151 & 126 & 3,9 & 2720 & 96 & 3,5 & $x_{1}^{2}=0,88 ; p>0,05^{*}$ \\
\hline
\end{tabular}

* Teste do Qui-quadrado para amostras independentes. 
Menezes CAS, Bittencourt AL, Mota E, Sherlock I, Ferreira J. Avaliação da parasitemia em mulheres portadoras de infecção pelo Trypanosoma cruzi durante e após a gestação. Revista da Sociedade Brasileira de Medicina Tropical 25:109-113, abr-jun, 1992.

Tabela 4 - Estudo comparativo dos resultaados obtidos nos $2^{\circ}$ e $3^{\circ}$ trimestres de gestação, em 75 mulheres com doença de Chagas crônica (grupo IV).

$\frac{\text { Segundo trimestre }}{\text { Total } \frac{\text { Positivos }}{\mathrm{n}^{\mathrm{o}} \%}}$

Terceiro trimestre

Total $\frac{\text { Positivos }}{\mathrm{n}^{\mathrm{o}} \%}$

Significância estatística

Xenos

realizados

144

28

19,4

168

36

21,4

$x_{1}^{2}=0,19 ; p>0,05^{*}$

Triatomíneos

examinados

1464

69

4,7

1838

91

4,9

$x_{1}^{2}=0,09 ; p>0,05 *$

* Teste do Qui-quadrado para amostras independentes.

Para avaliar a freqüência e os níveis de parasitemia no segundo e terceiro trimestres de gestação, analisaram-se os dados de um quarto grupo de 75 pacientes selecionadas dentre as 119 do grupo I, porque tiveram um ou mais xenos realizados no segundo e terceiro trimestres. Durante o terceiro trimestre, os parâmetros foram mais elevados em relação ao segundo trimestre, mas as diferenças não foram estatisticamente significantes (Tabela 4).

\section{DISCUSSÃO}

Oestudo comparativo da parasitemia, utilizando o mesmo grupo de mulheres, em um espaço de tempo inferior a 16 meses, permitiu excluir possíveis erros relacionados com a idade das pacientes e as cepas de $T$. cruzi. Experimentalmente, já se comprovou que diferentes cepas apresentam níveis parasitêmicos diversos e que a freqüência de positividade dos xenos varia de forma inversamente proporcional à idade dos pacientes ${ }^{16}$. A realização deste estudo em área não endêmica permitiu afastar casos recentes da infecção.

Os resultados deste trabalho indicam que ocorre, sem dúvida, um aumento da freqüência da parasitemia e dos níveis parasitêmicos durante a gestação, sendo mais acentuada no terceiro trimestre. No entanto, as diferenças observadas não foram muito grandes e, nos grupos menores (III e EV), não houve significância estatística. Acreditamos que isto seja devido ao pequeno número de pacientes que apresentou alterações da parasitemia na gravidez. Como já referido, apenas $17 \%$ das 119 pacientes estudadas tiveram dois ou mais xenos positivos durante a gravidez. Nestas mães, as diferenças entre as freqüências de xenos positivos e de triatomíneos infectados durante e após a gravidez foram muito acentuadas, sugerindo ter havido exacerbação da parasitemia pelo menos em algumas delas. Nestas pacientes, registrou-se um caso de transmissão congênita numa mulher, cujos quatro xenos realizados durante a gestação foram positivos (dado não publicado). Provavelmente, a transmissão transplacentária é favorecida pela maior freqüência da parasitemia e elevação dos níveis parasitêmicos.

A exacerbação da parasitemia na gravidez deve estar relacionada a alterações da imunidade celular que ocorrem durante este período ${ }^{3}$. É interessante referir que o agravamento da hanseníase na gravidez é mais acentuado no terceiro trimestre ${ }^{5}$. No quarto grupo das gestantes avaliadas neste trabalho, a freqüência da parasitemia e os níveis parasitêmicos foram também mais elevados neste período. No entanto, não há outras referências que indiquem maior susceptibilidade da gestante às infecções no terceiro trimestre ${ }^{3}$.

Poder-se-ia especular que a exacerbação da parasitemia em gestações sucessivas influenciaria desfavoravelmente a evolução da infecção materna. Este é um assunto que merece futura investigação. 
Menezes CAS, Bittencourt AL, Mota E, Sherlock I, Ferreira J. Avaliação da parasitemia em mulheres portadoras de infeç̧ão pelo Trypanosoma cruzi durante e após a gestação. Revista da Sociedade Brasileira de Medicina Tropical 25:109-113, abr-jun, 1992.

\section{SUMMARY}

This paper presents an evaluation of the parasitemic profiles of 119 women chronically infected with $\mathrm{T}$.cruzi. Xenodiagnosis (xenos) were applied during (465 xenos) and after pregnancy (363 xenos) in order to detect possible variations in parasitemia in these periods. The frequency of positive xenos was greater during than after gestation. Otherwise, the frequency of infected triatomines was wore elevated during pregnancy, indicating higher parasitemic levels in this period. Only 17\% of the studied women had two or more positivexenos during pregnancy. In these mothers the difference between the frequencies of positive xenos during and after gestation was high, suggesting the occurrence of exacerbation of infection at least in some women.

Key-words: Xenodiagnosis. Parasitemia in gestation. Chagas disease in pregnancy. Infectious diseases and pregnancy.

\section{AGRADECIMENTOS}

Agradecemos à colaboração do Prof. José Maria de Magalhães Neto, Diretor da Maternidade Climério de Oliveira, assim como dos funcionários do Serviço Pré-Natal desta Maternidade, do Laboratório Central do Estado da Bahia (LACEN) e doServiço de Entomologia do Centro de Pesquisas Gonçalo Muniz/FIOCRUZ.

\section{REFERÊNCIAS BIBLIOGRÁFICAS}

1. Andrade SG, Carvalho ML, Figueira RM. Carcterização morfológica e histopatológica de diferentes cepas do $T$. cruzi. Gazeta Médica da Bahia 70: 32-42, 1970.

2. Biocca A, Sequeira H. Prevalência de infeccion chagásica en embarazadas. In: Anais do $1^{\circ}$ Congresso Argentino de Parasitologia, Buenos Aires p. 43, 1972.

3. Brabin BJ. Epidemiology of infection in pregnancy. Reviews of Infectious Diseases 7: 579-603, 1985.

4. Carlier Y, Rivera MT, Truyens C, Puissont F, MilaneJ. Interactions between chronic murine T. cruzi infeccion and pregnancy: fetal growth retardation. American Journal of Tropical Medicine and Hygiene 37: 534-540, 1987.

5. Duncan ME, Person JM, Ridley DS, Melsom R, Bjune G. Pregnancy and leprosy: The consequences of alterations of cell mediated and humoral immunity during pregnancy and lactation. International Journal of Leprosy 50: 425-435, 1982.

6. Hoff R, Mott KE, Silva JEF, Menezes V, Hoff JN, Barrett TV, Sherlock IA. Prevalence of parasitemia and seroreactivity to Trypanosoma cruzi in a rural population of northeast Brazil. American Journal of
Tropical Medicine and Hygiene 28: 461-466, 1979.

7. Kohl S, Pickering LK, Frankel LS, Yaeger RG. Reactivation of Chagas'disease during therapy of acute lymphocytic leukemia. Cancer 50: 827-828, 1982.

8. Krampitz HE, Disko R. Retardation of parasitemia, prolongation of life or survival of lactating mice in ifections with Trypanosoma cruzi. Nature (London) 209: 526, 1966.

9. Reyes MB, Locca M, Muñoz P, Frasch AC. Fetal IgG specificities against Trypanosoma cruzi antigens in infected newborn. Proceedings of National Academy of Science (USA) 87: 2846-2850, 1990.

10. Storni P, Bolsi FL. Embarazo e parasitismo por Trypanosoma cruzi. Medicina (Buenos Aires) 39: 193-197, 1979.

11. Szarfman A, Urman J, Otalora A, Larguia A, Yanovsky JF. Specific agglutinins and immunoglobulin levels in congenital Chagas'infection. Medicina 35: 245-250, 1975.

12. Tanowitz HB, Morris SA, Factor SM, Weiss LM, Wittner M. Parasitic diseases of the heart I: Acute and chronic Chagas'disease. Cardiovascular Pathology 1: 7-15, 1992. 